\title{
Transcriptional profiles of Rhizobium vitis-inoculated and salicylic acid-treated 'Tamnara' grapevines based on microarray analysis
}

\author{
Youn Jung Choi $\cdot$ Hae Keun Yun
}

Received: 3 March 2016 / Revised: 3 March 2016 / Accepted: 9 March 2016

(c) Korean Society for Plant Biotechnology

\begin{abstract}
The transcriptional profiles of 'Tamnara' grapevine (Vitis labruscana L.) to Rhizobium vitis were determined using 12,000 gene oligonucleotide microarray chips constructed with 6,776 unigenes based on the EST sequencing. Among them, 95 clones were up-regulated more than three times and 90 were down-regulated more than 5-times in the $R$. vitis-inoculated grapevines relative to the control vines. Treatment of salicylic acid showed that 337 clones were upregulated and 52 clones were down regulated in grapevines. Microarray analysis, reverse transcription-polymer chain reaction, and slot blot hybridization analysis revealed that 5 , 14, and 64 clones were up-regulated and 10, 12, and 61 clones were down-regulated in wounded, salicylic acidtreated, and $R$. vitis-inoculated 'Tamnara' grapevine leaves, respectively. The expression patterns of $\beta-1,3$-glucanase, proline-rich protein, and lipoxygenase genes of 'Tamnara' moderately resistant to $R$. vitis were similar to those of resistant 'Concord' and 'Delaware' grapevines. However, chalcone synthase genes in 'Tamnara' grapevines showed similar expression patterns to susceptible grapevines 'Neomuscat' and 'Rizamat'. Further expression studies with various clones for each gene should be conducted to elucidate their roles in resistant responses against pathogens or other stimuli in grapevines. These results could provide better resources for understanding the mechanism of defense responses against crown gall disease and clues for identifying new genes that may play a role in defense against $R$. vitis in grapevines.
\end{abstract}

Y. J. Choi

National Institute for Horticultural and Herbal Science, Wanju 55365, Korea

H. K. Yun $(\bowtie)$

Department of Horticulture and Life Science, Yeungnam

University, Gyeonsan 38541, Korea

e-mail: haekeun@ynu.ac.kr
Keywords Grape, Gene expression, RT-PCR, Slot blot

\section{Introduction}

Grape (Vitis sp.) is attacted to a number of bacterial, fungal, and viral diseases like other plants (Pearson and Goheen 1998). Crown gall is a major disease responsible for severe reduction of yield and poor quality of fruit in grape production regions throughout the world, including Korea (Burr et al. 1998; Park et al. 2000). Chemical or biological attempts to control crown gall disease conducted to date have failed, with the exception of Agrobacterium radiobacter strain K84 of the biological control (Anand et al. 2008; Burr and Otten 1999). However, A. radiobacter strain $\mathrm{K} 84$ is only effective at controlling crown gall caused by nopaline-type strains of $A$. tumefaciens and A. rhizogenes, while it has no effect on crown galls induced by Rhizobium vitis in grapevines (Kerr 1980). Since effective agro-chemicals to control crown gall have not been released, development of novel grapevine cultivars resistant to crown gall is critical and will be a useful tool in protection of grapevines from disease (Burr et al. 1988; Park et al. 2000; Stover et al. 1997).

There has been continuous study of genes related to disease resistance and defense response of grapevines to fungal, bacterial, and viral pathogens, including crown gall disease through comparative genomics, transcriptomics and the genome wide identification analysis for useful genes and molecular markers (Burr et al. 1998 and 2003; Choi et al. 2008; Hur et al. 2015). However, development of disease resistant grapes based on molecular biology has been limited because of the relatively low amount of genetic and molecular information available regarding genotypes resistant to certain diseases. Although the entire genome of Vitis vinifera 'Pinot Noir' has been sequenced (The France-Italian Public Consortium for Grapevine 2007) and annotated, the functions of many genes must be still investigated. 
To develop new grape cultivars resistant to diseases, systematic monitoring of the mechanism of plant response and defense against pathogen attacks and more detailed functional studies of the selected differentially expressed genes (DEGs) are required. Microarray analysis, which can screen the expression patterns of many genes simultaneously in a single analysis, is considered a foundational technology capable of high-throughput and high-speed transcriptional profiling. Accordingly, this technique has various applications including unique gene identification and diagnostics of certain diseases (Schulze and Downward 2001; Stears 2003).

In this study, the gene expression patterns in response to $R$. vitis inoculation were investigated in $R$. vitis-inoculated, salicylic acid (SA)-treated, wounded, and untreated control leaves of 'Tamnara' grapevine which was bred in Korea (Park et al. 2004). Using microarray gene expression profiling, 6,776 unigenes of expressed sequence tag (EST)-based sequence were analyzed in grapevine leaves. The detailed expression patterns of selected up- and down-regulated genes in the microarray were confirmed by slot blot hybridization and semiquantitative reverse transcription-polymerase chain reaction (RT-PCR). Expression of DEGs was also analyzed in grapevines resistant and susceptible to crown gall in response to $R$. vitis inoculation.

\section{Materials and Methods}

Plant materials and treatments

Grapevines of 'Tamnara' (moderately resistant to crown gall disease), 'Delaware' and 'Concord' (resistant to crown gall disease), and 'Neomuscat' and 'Rizamat' (susceptible to crown gall disease) were grown in a greenhouse at $25^{\circ} \mathrm{C} \sim 30^{\circ} \mathrm{C}$ under natural light, then inoculated with $R$. vitis Cheonan 493 (Yun et al. 2003). Leaves were harvested at 1, 3, 6, 12, 24, 48, and $72 \mathrm{~h}$ after wound, SA treatment, and $R$. vitis inoculation, immediately frozen in liquid nitrogen, and stored at $-80^{\circ} \mathrm{C}$ until used for RNA extraction. All samples harvested from each treatment were used for RNA extraction, analysis of differential expression of cDNA, and RT-PCR analysis.

Microarray chip construction

A total of 6,776 unigenes were obtained from the 'Tamnara' grapevine cDNA library constructed after $R$. vitis inoculation and SA treatment. Microarray chips were constructed with 35 $-40 \mathrm{nt}$ of unigene based oligonucleotide. Gene specific oligonucleotides were arrayed onto a slide glass with an average of two replications and oligonucleotide microarray techniques were employed to detect $R$. vitis-responsive genes in 'Tamnara' grapevines.

RNA isolation and microarray hybridization

Total RNA was isolated from $R$. vitis-inoculated, SA-treated, and control grapevine leaves using the modified pine tree method of removing polysaccharides and phenolic compounds (Chang et al. 1993) with RNA extraction buffer consisting of $2 \%$ cetyltrimethylammonium bromide, $2 \%$ polyvinylpyrrolidone, 100 mM Tris- $\mathrm{HCl}$ ( $\mathrm{pH} 8.0), 25$ mMethylenediaminetetraacetic acid, $2 \mathrm{MNaCl}, 0.5 \mathrm{~g} \cdot \mathrm{L}^{-1}$ spermidine, and $2 \% \beta$-mercaptoethanol. To determine the SA and $R$. vitis-responsive genes, the oligonucleotide microarrays were hybridized with probes prepared from the total RNA of SA-treated, $R$. vitisinoculated, and control leaves.

The MessageAmp ${ }^{\text {TM }}$ II-Biotin Enhanced Single Round aRNA Amplification Kit (Ambion, Woodward Austin, TX, USA) is based on the RNA amplification protocol developed in the laboratory of James Eberwine (Van Gelder et al. 1990). Microarray hybridization was performed with $5 \mu \mathrm{g}$ of a labeled target sample per one CustomArray ${ }^{\mathrm{TM}}$ using a $12 \mathrm{~K}$ microarray hybridized and scanned PMT 500-700, pixel size of 5, focus position 130. Analyses were conducted using a GenePix 4000B microarray scanner (Axon Instruments, Union City, CA, USA). After data extraction, backgrounds for individual samples were calculated. One-way analysis of variance (ANOVA) and a t-test were applied to determine differentially expressed sets of genes across three experimental groups. Statistical significances were adjusted by BenjaminiHochberg FDR multiple-testing correction (Benjamini and Hochberg 1995). Complete linkage hierarchical clustering based on the Euclidean distances of samples was performed using the normalized significant genes. The patterns of expressed changes were analyzed for groups using the Avadis Prophetic Ver. 3.3 software (StrandGenomics, Bangalore, India, http://avadis. Strandgenomics.com/).

\section{Semiquantitative RT-PCR analysis}

Semiquantitative RT-PCR analysis was conducted using 95 up-regulated genes and 90 down-regulated genes. cDNAs was synthesized using a ReverTra-plus- ${ }^{\mathrm{TM}}$ High Fidelity RT-PCR Kit (Toyobo, PCR-501, Japan). PCR amplification was conducted by subjecting the samples to $94^{\circ} \mathrm{C}$ for $2 \mathrm{~min}$, followed by 30 cycles of $98^{\circ} \mathrm{C}$ for $10 \mathrm{~s}, 58^{\circ} \mathrm{C}$ for $30 \mathrm{~s}$ and $68^{\circ} \mathrm{C}$ for 1 min using KOD-Plus taq polymerase (Toyobo, KOD201, Japan). PCR amplification was conducted using primers 
specific for each gene and actin primers as an internal control under appropriate conditions.

RNA slot blot hybridization analysis

Total RNA $(5 \mu \mathrm{g})$ isolated from the leaves of grapevines was used for the RNA slot blot hybridization analysis. The RNA mixtures were denatured at $65^{\circ} \mathrm{C}$ for $10 \mathrm{~min}$, then blotted onto membranes using the Bio-Dot SF (BioRad). RNA samples were transferred and immobilized to Hybond- $\mathrm{N}^{+}$nylon membrane with UV-crosslinker. Hybridization, washing, detection, and exposure on X-ray film were performed as previously described.

\section{Results and Discussion}

Hierarchical clustering of the ESTs derived from 'Tamnara' grapevines

To obtain molecular profiles of responses to $R$. vitis in grapevines, the differential transcriptional profiles of $R$. vitis-inoculated and SA-treated grapevines were investigated using microarray analysis with 12,000 gene oligonucleotides. Samples for each treatment were harvested at multiple time points to screen for a large number of biochemical changes expected to occur after $R$. vitis-inoculation and SA-treatment. Genes showing induced and suppressed expression patterns in grapevines inoculated with $R$. vitis or treated with SA compared to untreated controls were selected as up- and down-regulated and used to validate expression analysis (Fig. 1).

As shown in the Venn diagram, 337 and 27 genes were induced more than two times by SA treatment and $R$. vitis inoculation, respectively (Fig. 1A). Among ten genes commonly up-regulated under both treatments, several were well-known defense-related genes, including proline-rich protein 1, leuco-anthocyanidin dioxgenase, cytosolic heat shock 70
A

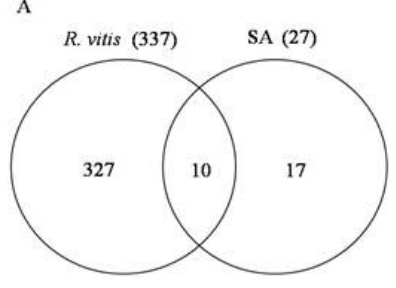

B

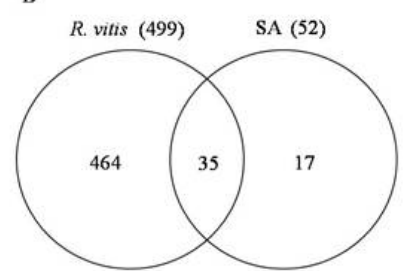

Fig. 1 Venn diagrams showing the numbers of overlapped and unique genes induced (A) and suppressed (B) more than twice in the level of their expressions by $R$. vitis inoculation and salicylic acid (SA) treatment. Results were based on the mean inductions of three replicates protein, and sarcosine oxidase family protein, while the functions of others were unknown. Similarly, among the ESTs of 'Tamnara' grapevines, 499 genes from $R$. vitisinoculated and 52 genes from SA-treated samples were down-regulated by more than two times, respectively (Fig. 1B). Additionally, 35 genes were suppressed by both treatments, which include genes encoding heavy metal-associated domaincontaining protein, $\mathrm{Zn}$ (C3HC4-type RING) finger family protein, putative WRKY transcription factors 4 and 30, MAP kinase-like protein, and hypothetical proteins. It was reported that several transcripts such as subtilisin-like protease, phenylalanine ammonia lyase (PAL), S-adenosylmethionine synthase, WD-repeat protein like, and J2P, were up-regulated in 'Regent' grapevine against the mildew (Figueiredo et al. 2013). Camparative analysis between resistant and susceptible grape cultivars to Pierce's disease showed that significant differences in transcripts including some of the PR proteins such as $\beta$-1,3-glucanase and chitinases and proline-rich proteins were shown at the stem tissues infected with Xylella fastidiosa (Lin et al. 2007). In this study, it was shown that expressions of various defense-related genes were differentially regulated in the grapevines by $R$. vitis infection and SA-treatment.

R. vitis and SA responsive DEGs with microarray analysis

Overall, 95 up-regulated cDNA clones showed expression that was up-regulate/d by more than 3-times, while 90 down-regulated clones showed decreases in expression of more than 5-times in $R$. vitis-inoculated grapevines relative to untreated samples (Tables 1 and 2). Arabidopsis thaliana seed imbibition 2 (ATSI2) hydrolyzing O-glycosyl compound gene and btb and taz domain protein related genes were highly up-regulated. Conversely, cell wall protein, expansin, and endosperm specific protein genes were significantly suppressed. Additionally, the transcriptional levels of defense, signal transduction, active oxygen related genes such as methallothionine-like protein, LRR domains, and PR protein, as well as transcription factors such as MYB transcription factors were highly activated. These results were consistent with those of ESTs from $R$. vitis inoculated 'Tamnara' grapevines (Choi et al. 2010).

As shown in the Tables 1 and 2, some genes showed antagonistic expression patterns between induced genes of $R$. vitis-inoculated and SA-treated grapevines. The genes activated by $R$. vitis inoculation might be mediated by jasmonic acid (JA) or ethylene. The expression of 95 up-regulated genes was suppressed by $R$. vitis inoculation in SA-treated grapevine leaves. In contrast, the expression of 90 genes down-regulated by $R$. vitis inoculation increased in response 
Table 1 Genes in 'Tamnara' grapevines up-regulated in response to $R$. vitis inoculation and SA treatment

\begin{tabular}{|c|c|c|c|c|c|}
\hline \multirow{2}{*}{$\begin{array}{c}\text { Gene No. in } \\
\text { EPP163JWAA } \\
\text { series } \\
\end{array}$} & \multirow{2}{*}{\multicolumn{2}{|c|}{$\begin{array}{l}\text { No. of slot blot, } \\
\text { RT-PCR }\end{array}$}} & \multirow{2}{*}{ Putative function } & \multicolumn{2}{|c|}{ Ratio of signal intensity } \\
\hline & & & & R. vitis & SA \\
\hline $12 \mathrm{C} 000256$ & B9 & U1 & ATSI 2 hydrolyzing O-glycosyl compounds yabby 15 protein & $15.9 \pm 0.49$ & $-14.2 \pm 0.44$ \\
\hline $12 \mathrm{~S} 013353$ & $\mathrm{~F} 4$ & $\mathrm{U} 2$ & Btb and taz domain protein & $11.2 \pm 0.85$ & $-4.2 \pm 0.27$ \\
\hline $12 \mathrm{C} 000750$ & $\mathrm{~F} 2$ & U3 & Metallothionein-like protein & $10.3 \pm 0.63$ & $-6.0 \pm 0.79$ \\
\hline $12 \mathrm{C} 000845$ & D7 & $\mathrm{U} 4$ & LOX & $9.3 \pm 0.52$ & $-11.1 \pm 0.66$ \\
\hline $12 \mathrm{C} 001644$ & B6 & U5 & Dark inducible 10 hydrolyzing O-glycosyl compounds & $7.6 \pm 0.46$ & $-7.8 \pm 0.36$ \\
\hline $12 \mathrm{~S} 000483$ & $\mathrm{H} 3$ & U6 & No hit & $7.6 \pm 0.70$ & $-3.5 \pm 0.81$ \\
\hline $12 \mathrm{~S} 008848$ & $\mathrm{H} 1$ & U7 & Hypothetical protein & $7.4 \pm 0.19$ & $-3.1 \pm 0.71$ \\
\hline $12 \mathrm{~S} 003419$ & A7 & U8 & Glycosyl hydrolase family 1 protein & $7.0 \pm 0.15$ & $-4.8 \pm 0.25$ \\
\hline $12 \mathrm{~S} 007850$ & G6 & U9 & CHS & $6.8 \pm 0.94$ & $-4.5 \pm 0.72$ \\
\hline $12 \mathrm{~S} 003388$ & A3 & U10 & Glycosyl hydrolase family 1 protein & $6.5 \pm 0.07$ & $-4.7 \pm 0.21$ \\
\hline $12 \mathrm{~S} 009383$ & F5 & U11 & Aspartyl protease family protein & $6.4 \pm 0.57$ & $-4.2 \pm 0.63$ \\
\hline $12 \mathrm{~S} 005565$ & $\mathrm{~B} 2$ & U12 & Glucose-6-phosphate translocator & $6.3 \pm 0.18$ & $-5.5 \pm 0.31$ \\
\hline $12 \mathrm{~S} 002570$ & $\mathrm{~B} 10$ & U13 & Limonoid udp-lucosyltransferase & $6.2 \pm 0.26$ & $-3.6 \pm 0.39$ \\
\hline $12 \mathrm{~S} 002915$ & B3 & U14 & Cytochrome P450 & $6.2 \pm 0.17$ & $-4.2 \pm 0.32$ \\
\hline $12 \mathrm{~S} 009437$ & A12 & U15 & ATSI 2 hydrolyzing O-glycosyl compounds & $6.0 \pm 0.36$ & $-7.3 \pm 0.16$ \\
\hline $12 \mathrm{~S} 006649$ & $\mathrm{C} 4$ & U16 & Isoamylase isoform 3 & $6.0 \pm 0.12$ & $-5.2 \pm 0.44$ \\
\hline $12 \mathrm{~S} 000876$ & D2 & U17 & Cytochrome p450 & $6.0 \pm 0.43$ & $-4.9 \pm 0.33$ \\
\hline $12 \mathrm{~S} 010715$ & G3 & U18 & Cytochrome $\mathrm{p} 450$ & $5.9 \pm 0.69$ & $-3.4 \pm 0.58$ \\
\hline $12 \mathrm{~S} 012744$ & E11 & U19 & Proline-rich cell wall protein & $5.9 \pm 0.49$ & $-2.9 \pm 0.53$ \\
\hline $12 \mathrm{~S} 005981$ & A5 & $\mathrm{U} 20$ & Organic cation transporter & $5.8 \pm 0.19$ & $-5.0 \pm 0.22$ \\
\hline $12 \mathrm{~S} 002410$ & D4 & $\mathrm{U} 21$ & Thaumatin-like protein & $5.8 \pm 0.60$ & $-6.9 \pm 0.57$ \\
\hline $12 \mathrm{~S} 009693$ & D11 & $\mathrm{U} 22$ & Starch phosphorylase & $5.8 \pm 0.44$ & $-4.2 \pm 0.34$ \\
\hline $12 \mathrm{C} 000042$ & G2 & $\mathrm{U} 23$ & Myb transcription factor & $5.7 \pm 0.54$ & $-4.1 \pm 0.70$ \\
\hline 12S013702 & A6 & $\mathrm{U} 24$ & Aldehyde dehydrogenase & $5.7 \pm 0.16$ & $-6.0 \pm 0.27$ \\
\hline $12 \mathrm{C} 001360$ & $\mathrm{C} 1$ & $\mathrm{U} 25$ & Protein & $5.6 \pm 0.33$ & $-6.0 \pm 0.38$ \\
\hline $12 \mathrm{~S} 011414$ & E4 & U26 & GST & $5.6 \pm 0.11$ & $-3.9 \pm 0.56$ \\
\hline $12 \mathrm{~S} 000490$ & $\mathrm{~A} 2$ & $\mathrm{U} 27$ & Mate efflux family expressed & $5.5 \pm 0.15$ & $-3.5 \pm 0.10$ \\
\hline $12 \mathrm{~S} 012305$ & B12 & $\mathrm{U} 28$ & Hypothetical protein & $5.5 \pm 0.25$ & $-4.2 \pm 0.37$ \\
\hline $12 \mathrm{~S} 006861$ & $\mathrm{C} 5$ & $\mathrm{U} 29$ & Reductase 1 & $5.3 \pm 0.25$ & $-2.7 \pm 0.30$ \\
\hline $12 \mathrm{~S} 006594$ & A11 & U30 & Molybdenum cofactor sulfurase & $4.9 \pm 0.22$ & $-6.2 \pm 0.28$ \\
\hline $12 \mathrm{~S} 007535$ & A10 & U31 & Glyoxalase i & $4.6 \pm 0.13$ & $-2.3 \pm 0.22$ \\
\hline $12 \mathrm{~S} 005609$ & D8 & U32 & Expansin-like protein & $4.6 \pm 0.14$ & $-3.7 \pm 0.46$ \\
\hline $12 \mathrm{C} 000943$ & D1 & U33 & Ef-1 a & $4.5 \pm 0.40$ & $-2.7 \pm 0.26$ \\
\hline 12S011005 & $\mathrm{C} 3$ & U34 & Alkaline a galactosidase & $4.4 \pm 0.35$ & $-4.7 \pm 0.33$ \\
\hline $12 \mathrm{~S} 000980$ & D10 & U35 & Fatty acid hydroperoxide lyase & $4.4 \pm 0.29$ & $-3.6 \pm 0.39$ \\
\hline $12 \mathrm{~S} 002559$ & B7 & U36 & Af303396_1udp-glucosyltransferase hra25 & $4.4 \pm 0.28$ & $-2.9 \pm 0.16$ \\
\hline $12 \mathrm{~S} 010472$ & B11 & U37 & Tpa:gid1-like gibberellin receptor & $4.4 \pm 0.33$ & $-3.0 \pm 0.06$ \\
\hline $12 \mathrm{~S} 006784$ & F8 & U38 & Glucose acyltransferase & $4.3 \pm 0.50$ & $-3.7 \pm 0.35$ \\
\hline $12 \mathrm{~S} 011826$ & F12 & U39 & Ethephon-induced protein & $4.3 \pm 0.49$ & $-3.1 \pm 0.50$ \\
\hline $12 \mathrm{~S} 005921$ & $\mathrm{C} 7$ & $\mathrm{U} 40$ & Fibrillin & $4.3 \pm 0.25$ & $-2.9 \pm 0.28$ \\
\hline $12 \mathrm{~S} 009053$ & E6 & U41 & Condensation domain-containing protein & $4.2 \pm 0.46$ & $-3.0 \pm 0.11$ \\
\hline $12 \mathrm{C} 000227$ & E3 & U42 & Basic helix-loop-helixfamily protein & $4.2 \pm 0.14$ & $-2.7 \pm 0.44$ \\
\hline $12 \mathrm{C} 000359$ & E1 & U43 & Sucrose synthase & $4.1 \pm 0.21$ & $-3.6 \pm 0.22$ \\
\hline $12 \mathrm{~S} 010980$ & A9 & U44 & Pyrroline-5-carboxylate synthetase & $4.1 \pm 0.44$ & $-5.7 \pm 0.34$ \\
\hline $12 \mathrm{~S} 002233$ & E12 & U45 & Glyceraldehyde-3-phosphate dehydrogenase & $4.1 \pm 0.48$ & $-3.5 \pm 0.28$ \\
\hline $12 \mathrm{C} 001111$ & G5 & U46 & Chalcone-flavanone isomerase family expressed & $4.1 \pm 0.63$ & $-2.9 \pm 0.54$ \\
\hline $12 \mathrm{~S} 011777$ & $\mathrm{H} 10$ & U47 & Abscisic acid responsive elements-binding factor & $4.0 \pm 0.92$ & $-2.3 \pm 0.15$ \\
\hline 12S009704 & A4 & U48 & Hypothetical protein & $4.0 \pm 0.14$ & $-3.0 \pm 0.16$ \\
\hline $12 \mathrm{C} 001087$ & H11 & U49 & Methionine gamma-lyase & $3.9 \pm 0.30$ & $-2.6 \pm 0.92$ \\
\hline $12 \mathrm{~S} 002496$ & $\mathrm{H} 2$ & U50 & Protein kinase & $3.8 \pm 0.33$ & $-3.0 \pm 0.60$ \\
\hline
\end{tabular}


Table 2 Genes in 'Tamnara' grapevines down-regulated in response to $R$. vitis inoculation and SA treatment

\begin{tabular}{|c|c|c|c|c|c|}
\hline \multirow{3}{*}{$\begin{array}{c}\text { Gene No. in } \\
\text { EPP163JWAA } \\
\text { Series } \\
12 \mathrm{C} 001593\end{array}$} & \multirow{2}{*}{\multicolumn{2}{|c|}{$\begin{array}{c}\text { No. of slot blot, } \\
\text { RT-PCR }\end{array}$}} & \multirow{2}{*}{ Putative function } & \multicolumn{2}{|c|}{ Ratio of signal intensity } \\
\hline & & & & \multirow{2}{*}{$\frac{R . \text { vitis }}{-50.2 \pm 0.87}$} & \multirow{2}{*}{$\frac{\text { SA }}{36.1 \pm 0.56}$} \\
\hline & D8 & D1 & Cell wall protein & & \\
\hline $12 \mathrm{~S} 000426$ & A2 & D2 & Expansin & $-45.6 \pm 0.20$ & $32.6 \pm 0.32$ \\
\hline $12 \mathrm{~S} 005616$ & A10 & D3 & At5g25460 f18g18_200 & $-33.9 \pm 0.47$ & $32.6 \pm 0.33$ \\
\hline $12 \mathrm{C} 000040$ & F5 & D4 & Cytochrome $\mathrm{C}$ oxidase polypeptide vc & $-21.7 \pm 0.94$ & $16.0 \pm 0.78$ \\
\hline $12 \mathrm{C} 000966$ & E7 & D5 & Endosperm specific & $-21.7 \pm 0.82$ & $16.1 \pm 0.65$ \\
\hline $12 \mathrm{C} 000783$ & B1 & D6 & Protease inhibitor seed storage lipid transfer protein & $-21.6 \pm 0.40$ & $15.2 \pm 0.41$ \\
\hline $12 \mathrm{C} 000998$ & E4 & D7 & Protein & $-18.1 \pm 0.83$ & $17.4 \pm 0.59$ \\
\hline 12S003758 & $\mathrm{F} 1$ & D8 & En/Spm-like transposon protein & $-17.9 \pm 0.92$ & $15.1 \pm 0.48$ \\
\hline $12 \mathrm{C} 001094$ & F12 & D9 & Meiosis 5 & $-17.8 \pm 1.09$ & $13.8 \pm 0.89$ \\
\hline $12 \mathrm{~S} 013145$ & A9 & D10 & Proline-rich protein apg isolog & $-16.4 \pm 0.20$ & $11.3 \pm 0.37$ \\
\hline $12 \mathrm{~S} 010548$ & B12 & D11 & DNA heat shock N-terminal domain-containing protein & $-15.9 \pm 0.39$ & $12.3 \pm 0.49$ \\
\hline $12 \mathrm{C} 000058$ & G6 & D12 & Acid phosphatase & $-15.4 \pm 1.42$ & $9.4 \pm 0.57$ \\
\hline $12 \mathrm{C} 000034$ & A6 & D13 & Expansin & $-14.9 \pm 0.27$ & $17.3 \pm 0.19$ \\
\hline $12 \mathrm{C} 001244$ & B6 & D14 & Heavy metal-associated domain-containing protein & $-14.7 \pm 0.31$ & $5.5 \pm 0.40$ \\
\hline $12 \mathrm{C} 000247$ & A5 & D15 & SA-induced fragment 1 protein & $-13.8 \pm 0.21$ & $13.4 \pm 0.27$ \\
\hline $12 \mathrm{~S} 011527$ & F2 & D16 & Cold induced & $-13.6 \pm 0.84$ & $5.1 \pm 0.17$ \\
\hline $12 \mathrm{C} 000203$ & A4 & D17 & Rho GDP-dissociation inhibitor 1 & $-13.4 \pm 0.26$ & $7.8 \pm 0.20$ \\
\hline $12 \mathrm{C} 001631$ & E2 & D18 & Proline rich protein 2 & $-13.1 \pm 0.77$ & $11.1 \pm 0.26$ \\
\hline $12 \mathrm{~S} 009428$ & $\mathrm{~B} 10$ & D19 & Hypothetical protein & $-13.1 \pm 0.48$ & $12.5 \pm 0.15$ \\
\hline $12 \mathrm{C} 000933$ & A7 & D20 & Fatty acid elongase & $-12.8 \pm 0.30$ & $9.7 \pm 0.21$ \\
\hline 12S005808 & A1 & D21 & Proline-rich protein & $-10.8 \pm 0.06$ & $14.1 \pm 0.20$ \\
\hline $12 C 000939$ & D5 & $\mathrm{D} 22$ & $\beta$-Ketoacyl-synthase & $-10.7 \pm 0.25$ & $4.5 \pm 0.52$ \\
\hline $12 \mathrm{C} 001125$ & D10 & D23 & Xyloglucan endotransglycosylase & $-10.6 \pm 0.64$ & $10.8 \pm 0.43$ \\
\hline $12 \mathrm{~S} 011610$ & D9 & D24 & Hypothetical protein & $-10.6 \pm 0.23$ & $7.8 \pm 0.56$ \\
\hline $12 \mathrm{~S} 008346$ & G7 & D25 & No hit & $-10.0 \pm 1.35$ & $12.0 \pm 0.57$ \\
\hline $12 \mathrm{~S} 008268$ & F11 & D26 & Tonoplast membrane integral protein $4-4$ & $-9.9 \pm 0.76$ & $5.2 \pm 0.48$ \\
\hline 12S011177 & D3 & D27 & Hypothetical protein & $-9.7 \pm 0.49$ & $7.5 \pm 0.19$ \\
\hline $12 \mathrm{C} 000816$ & C6 & D28 & Chloroplast chlorophyll $\mathrm{a} / \mathrm{b}$ binding protein & $-9.7 \pm 0.48$ & $14.7 \pm 0.22$ \\
\hline $12 \mathrm{C} 000797$ & A8 & D29 & Chloroplast chlorophyll $\mathrm{a} / \mathrm{b}$ binding protein & $-9.6 \pm 0.30$ & $10.5 \pm 0.34$ \\
\hline $12 \mathrm{~S} 005860$ & E5 & D30 & CCHC-type integrase & $-9.5 \pm 0.28$ & $3.6 \pm 0.65$ \\
\hline $12 \mathrm{C} 000927$ & G1 & D31 & Protein binding protein & $-8.8 \pm 0.74$ & $5.8 \pm 0.51$ \\
\hline $12 \mathrm{~S} 008817$ & $\mathrm{C} 3$ & D32 & LRR protein & $-8.5 \pm 0.15$ & $6.2 \pm 0.39$ \\
\hline $12 \mathrm{C} 001369$ & D4 & D33 & Transferase family protein & $-8.4 \pm 0.24$ & $10.3 \pm 0.52$ \\
\hline $12 \mathrm{~S} 001577$ & $\mathrm{C} 1$ & D34 & No hit & $-8.4 \pm 0.18$ & $3.1 \pm 0.34$ \\
\hline $12 \mathrm{~S} 003449$ & A3 & D35 & Mee60 (maternal effect embryo arrest 60) & $-8.3 \pm 0.21$ & $11.9 \pm 0.21$ \\
\hline $12 \mathrm{C} 001193$ & B9 & D36 & Yabby 15 protein & $-8.1 \pm 0.28$ & $6.8 \pm 0.36$ \\
\hline 12S009302 & $\mathrm{G} 2$ & D37 & Aspartyl protease family protein & $-8.0 \pm 0.74$ & $5.7 \pm 0.73$ \\
\hline $12 \mathrm{~S} 011056$ & B11 & D38 & At3g15630 msj11_3 & $-7.9 \pm 0.25$ & $3.0 \pm 0.25$ \\
\hline $12 \mathrm{C} 001299$ & E1 & D39 & Endo- $\beta$-glucanase precursor & $-7.8 \pm 0.46$ & $4.7 \pm 0.46$ \\
\hline $12 \mathrm{~S} 013533$ & $\mathrm{H} 2$ & D40 & Expansin-like protein a & $-7.7 \pm 0.42$ & $2.2 \pm 0.66$ \\
\hline $12 \mathrm{~S} 013059$ & G5 & D41 & Thaumatin-like protein & $-7.7 \pm 1.01$ & $7.0 \pm 0.62$ \\
\hline $12 \mathrm{C} 000786$ & F7 & D42 & Tonoplast intrinsic protein & $-7.6 \pm 0.63$ & $5.6 \pm 0.49$ \\
\hline 12S006978 & $\mathrm{C} 10$ & D43 & WRKY transcription factor 10 & $-7.5 \pm 0.39$ & $5.3 \pm 0.32$ \\
\hline $12 \mathrm{C} 000038$ & B8 & D44 & Hypothetical protein & $-7.5 \pm 0.32$ & $4.8 \pm 0.18$ \\
\hline $12 \mathrm{C} 001095$ & G10 & D45 & a Tubulin 1 & $-7.3 \pm 1.16$ & $3.9 \pm 0.58$ \\
\hline 12S010793 & F10 & D46 & At5g44130 mln1_5 & $-7.2 \pm 0.60$ & $5.8 \pm 0.62$ \\
\hline $12 \mathrm{C} 000621$ & E8 & D47 & Chitinase-like protein & $-7.2 \pm 0.58$ & $5.5 \pm 0.29$ \\
\hline $12 \mathrm{C} 000406$ & G3 & D48 & Glutamine synthetase & $-7.2 \pm 0.76$ & $4.9 \pm 0.66$ \\
\hline $12 \mathrm{C} 000508$ & B7 & D49 & Nucleoid DNA-binding protein cnd 41 & $-7.1 \pm 0.18$ & $6.7 \pm 0.35$ \\
\hline $12 \mathrm{~S} 009795$ & $\mathrm{C} 5$ & D50 & Calmodulin-like protein & $-7.0 \pm 0.29$ & $5.2 \pm 0.35$ \\
\hline
\end{tabular}


Table 3 Defense-related cDNA responsive to $R$. vitis inoculation and SA treatment in 'Tamnara' grapevines

\begin{tabular}{|c|c|c|c|}
\hline \multirow{2}{*}{ Gene } & \multirow{2}{*}{ Putative function/homology } & \multicolumn{2}{|c|}{ Ratio of signal intensity } \\
\hline & & R. vitis & SA \\
\hline \multirow[t]{6}{*}{ Defense-related } & $\beta-1,3$-Glucanase & $-3.4 \pm 0.36$ & $-1.4 \pm 0.38$ \\
\hline & Chitinase & $-4.4 \pm 0.25$ & $-1.2 \pm 0.26$ \\
\hline & Basic endochitinase precursor & $2.3 \pm 0.37$ & $-1.7 \pm 0.32$ \\
\hline & Chitinase III & $-2.6 \pm 0.51$ & $-1.4 \pm 0.66$ \\
\hline & Thaumatin-like protein & $5.8 \pm 0.60$ & $-1.3 \pm 0.24$ \\
\hline & Chalcone synthase & $6.8 \pm 0.94$ & $1.5 \pm 1.11$ \\
\hline \multirow[t]{2}{*}{ Signal transduction } & LOX & $9.3 \pm 0.52$ & $-1.2 \pm 0.69$ \\
\hline & NBS LRR-containing protein & $3.5 \pm 0.73$ & $1.2 \pm 0.81$ \\
\hline \multirow[t]{4}{*}{ Active oxygen related } & Catalase & $2.5 \pm 0.71$ & $-1.1 \pm 0.70$ \\
\hline & Glutathione peroxidase & $3.2 \pm 0.19$ & $1.1 \pm 0.42$ \\
\hline & Glutathione S-transferase & $5.6 \pm 0.11$ & $1.4 \pm 0.56$ \\
\hline & Ascorbate peroxidase & $1.8 \pm 0.25$ & $1.1 \pm 0.23$ \\
\hline \multirow[t]{2}{*}{ Secondary metabolites } & Cytochrome P450 & $6.0 \pm 0.43$ & $-4.9 \pm 0.33$ \\
\hline & Cytochrome $\mathrm{C}$ oxidase subunit $\mathrm{Vb}$ & $1.8 \pm 0.21$ & $1.1 \pm 0.33$ \\
\hline \multirow[t]{2}{*}{ Abiotic stress-related } & Small heat shock protein & $3.5 \pm 0.29$ & $1.3 \pm 0.35$ \\
\hline & Heat shock protein & $3.7 \pm 0.18$ & $1.6 \pm 0.32$ \\
\hline Cell wall fortification & Proline-rich cell wall protein & $5.9 \pm 0.49$ & $2.0 \pm 0.43$ \\
\hline \multirow[t]{2}{*}{ Transcription factors } & WRKY transcription factor & $1.7 \pm 0.21$ & $-1.1 \pm 0.26$ \\
\hline & MYB transcription factor & $5.8 \pm 0.54$ & $1.4 \pm 0.84$ \\
\hline
\end{tabular}

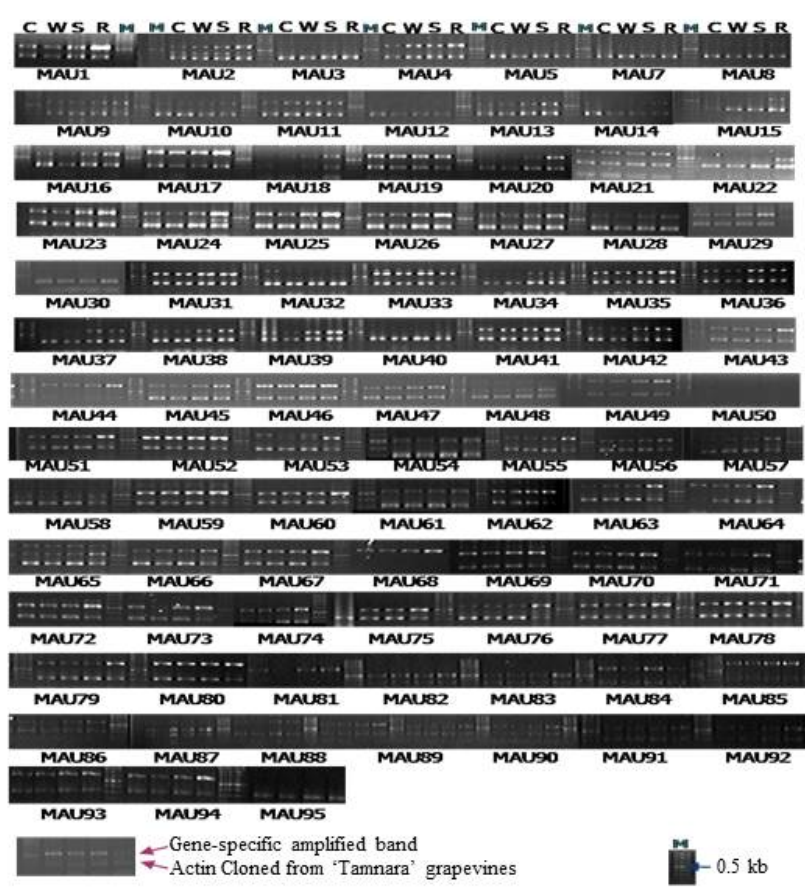

Fig. 2 Semiquantitative RT-PCR analysis of 95 highly up-regulated genes in 'Tamnara' grapevines. cDNA samples were amplified with a ReverTra-plus ${ }^{\text {TM }}$-High Fidelity RT-PCR Kit. MAU: up-regulated in microarray analysis; C, control; W, wound; S, SA treatment; $\mathrm{R}, R$. vitis inoculation; $\mathrm{M}$, size marker

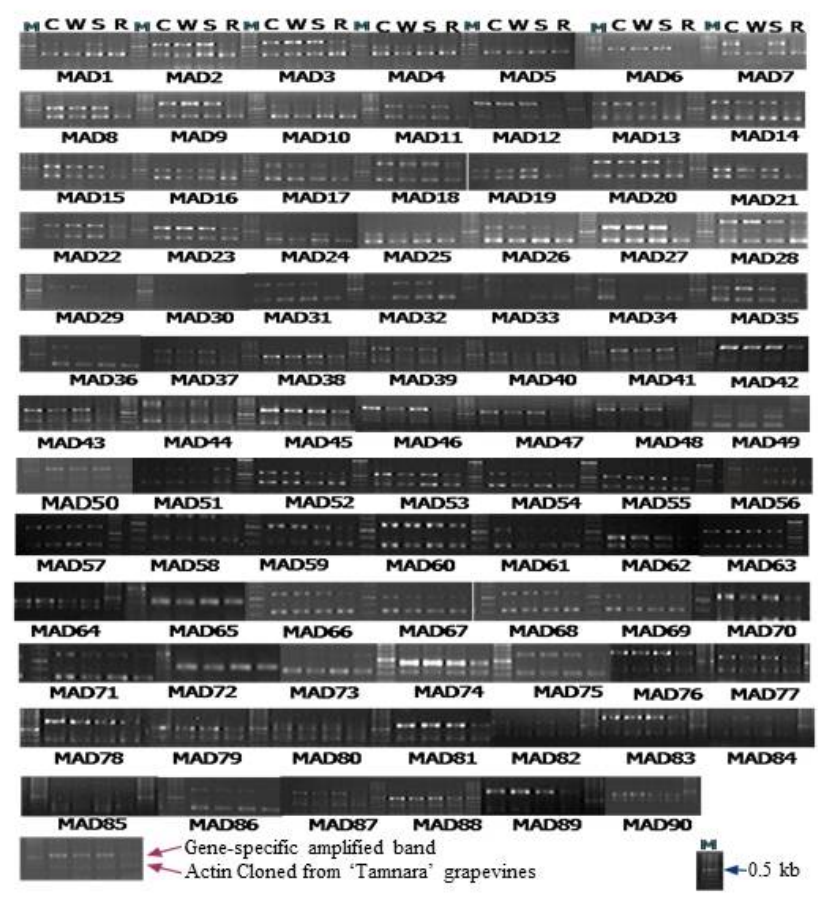

Fig. 3 Semiquantitative RT-PCR analysis of 90 highly downregulated genes in 'Tamnara' grapevines. cDNA samples were amplified with a ReverTra-plus ${ }^{\mathrm{TM}}$-High Fidelity RT-PCR Kit. MAD: down-regulated in microarray analysis; C, control; W, wound; S, SA treatment; $\mathrm{R}, R$. vitis inoculation; $\mathrm{M}$, size marker 
to SA treatment. When $R$. vitis attacks the vines, highly induced JA-dependent responses might suppress the gene expression involved SA-dependent defense pathway due to pathway crosstalk by $R$. vitis. Antagonisms between JA or ethylene and SA signaling have been extensively studied (Dong 1998; Kunkel and Brooks 2002). A large number of genes, includingc lipoxygenase (LOX), lipid transferase, and genes related to secondary metabolisms (Creelman and Mullet 1997; Lin et al. 2007) that were involved in JA biosynthesis, have been shown to respond to wounding and $R$. vitis attack in grapevines. The crosstalk among a number of signaling molecules appears to be related to controlling defense systems in response to pathogen attacks in grapevines as in other plants (Shah 2003).

Genes in response to $R$. vitis, SA, and wound in 'Tamnara' grapevines

Transcriptional profiling in response to $R$. vitis with the 6,776 unigenes obtained from the $R$. vitis-inoculated and SA-treated 'Tamnara' grapevine cDNA library was conducted by use of gene specific oligonucleotide microarray chips. cDNA contigs responsive to $R$. vitis inoculation and SA treatment could be categorized into seven groups encoding proteins involved in defense, defense signaling, oxidative burst, secondary metabolism, abiotic stress, cell wall fortification, and transcription factors (Table 3). The expression level of 95 up-regulated and 90 down-regulated ESTs was confirmed by semiquantitative RT-PCR (Fig. 2 and 3) and RNA slot blot hybridization analysis (Fig. 5 and 6). Confirmation using the three expression profiling methods revealed that 5,14 , and 64 cDNAs were up-regulated by wound, SA-treatment, and $R$. vitis inoculation, while 10 , 12 , and 61 were down-regulated by each treatment, respectively (Table 4).

As shown in the Venn diagram (Fig. 4A), 50 cDNAs, including ATSI protein 2 hydrolyzing O-glycosyl compounds yabby 15 protein, CHS, cytochrome P450, thaumatin-like protein, GST, and LRR containing protein, were specifically activated by $R$. vitis inoculation in 'Tamnara' grapevine leaves. However, no cDNA was shown to be up-regulated by wound or SA treatment in grapevines. Five genes, LOX, aspartyl protease family protein, heavy metal-associated domaincontaining protein, tyrosine aminotransferase, and one with unknown function, were commonly induced by $R$. vitis inoculation, wound, and SA treatments. Similarly, 1, 4, and 48 cDNAs were down-regulated by wound, SA treatment and $R$. vitis inoculation, respectively (Fig. 4B). Expansin, cytochrome $\mathrm{C}$ oxidase polypeptide vc, seed storage lipid transfer protein, meiosis 5 , SA-induced fragment 1 protein,
WRKY transcription factor 10, chitinase-like protein, and Zn finger (gata type) family proteins were specifically downregulated by $R$. vitis inoculation. Four genes such as lipid transfer protein, extensin-like protein, and two proteins with unknown function were commonly inhibited under both $R$. vitis inoculation and SA treatment (Table 5). More detailed functional studies should be conducted to determine if they are involved in defense mechanisms. It has been reported that PR genes were activated by endo- and exogenous SA treatments and pathogen attacks in many plants (Malamy 1990). In this study, genes involved in plant defense responses such as thaumatin-like protein, chalcone synthase (CHS), and LOX were induced by $R$. vitis inoculation and SA treatment.

In this study, some genes such as $\beta-1,3$-glucanase and chitinase III responded similarly to $R$. vitis inoculation and SA treatment. Using the GeneFishing and RACE technology, the full-length cDNA of several PR genes including $\beta-1,3$ glucanase, PR 10, and thaumatin-like proteins expressed specifically by $R$. vitis inoculation and SA treatment were cloned from the grapevine leaves (Choi et al. 2008). Cheong et al. (2002) reported that various genes such as LOX, catalase

Table 4 Genes specifically expressed in response to wound, SA treatment, and R. vitis inoculation with microarray, RT-PCR, and slot blot hybridization analyses in 'Tamnara' grapevines

\begin{tabular}{lccc}
\hline A. Up-regulated genes & & & \\
\hline \multicolumn{1}{c}{ Confirming method } & Wound & SA & R. vitis \\
\hline Microarray and slot blot & 78 & 37 & 82 \\
Microarray and RT-PCR & 7 & 32 & 73 \\
Microarray, slotblot, and RT-PCR & 5 & 14 & 64 \\
\hline
\end{tabular}

B. Down-regulated genes

\begin{tabular}{lccc}
\hline \multicolumn{1}{c}{ Confirming method } & Wound & SA & $R$. vitis \\
\hline Microarray and slot blot & 70 & 64 & 71 \\
Microarray and RT-PCR & 14 & 16 & 74 \\
Microarray, slotblot, and RT-PCR & 10 & 12 & 61 \\
\hline
\end{tabular}
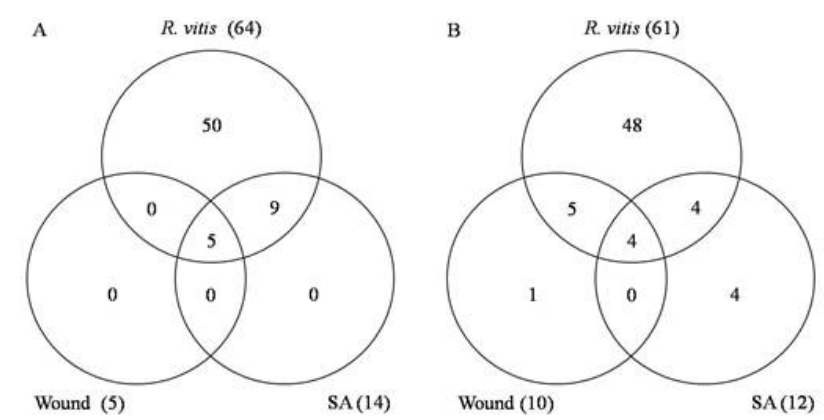

Fig. 4 Venn diagram of DEGs upregulated (A) and down-regulated (B) in 'Tamnara' grapevines responsive to $R$. vitis inoculation, wound, and SA treatment 
(CAT), glutathione $S$-transferase (GST), cytochrome P450, and WRKY, as well as MYB transcription factors were activated by wound stress in grapevines. A number of components in their signaling pathways appear to share the response against pathogen infection and wounding stress (Cheong et al. 2002; Maleck and Dietrich 1999). Accordingly, further functional analysis is required to determine the expression dynamics of selected genes in response to mechanical wounding, $R$. vitis inoculation, and SA treatment.
Semiquantitative RT-PCR and slot blot hybridization analysis of cDNA

The expression level of 95 up-regulated and 90 downregulated ESTs was confirmed by semiquantitative RT-PCR (Fig. 2 and 3) and RNA slot blot hybridization analysis (Fig. 5 and 6). Confirmation using the three expression profiling methods revealed that 5,14 , and $64 \mathrm{cDNAs}$ were up-regulated by wound, SA treatment, and $R$. vitis inoculation, while 10 ,

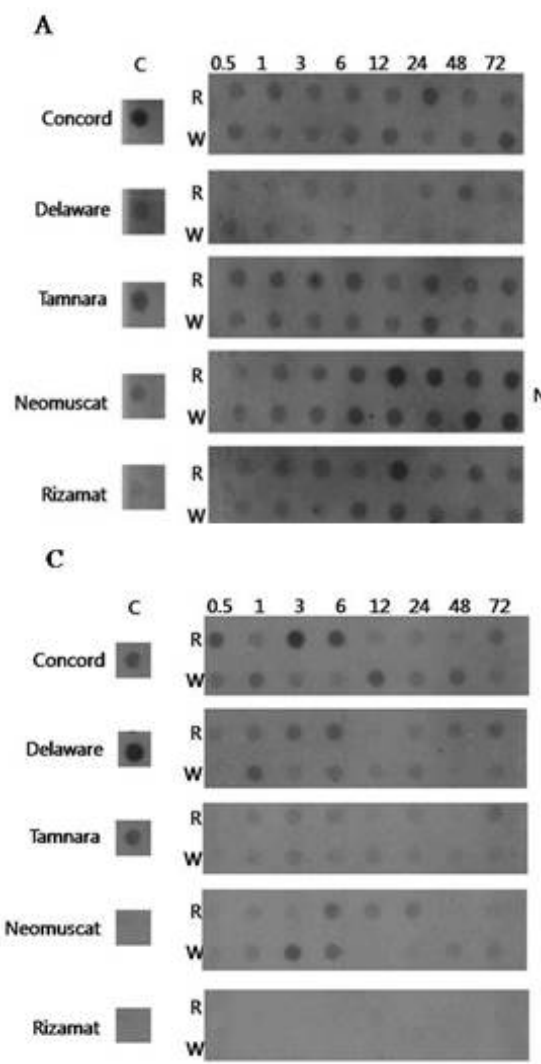

B

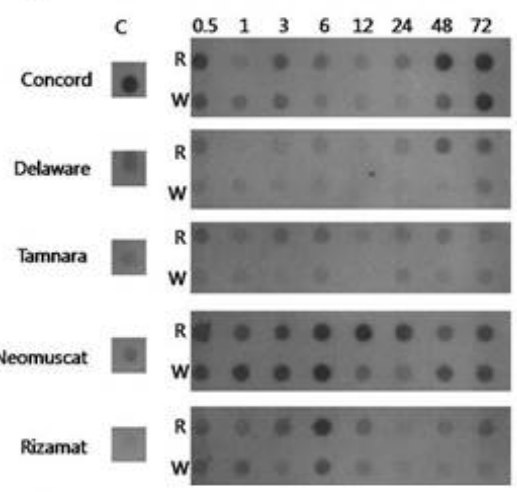

D

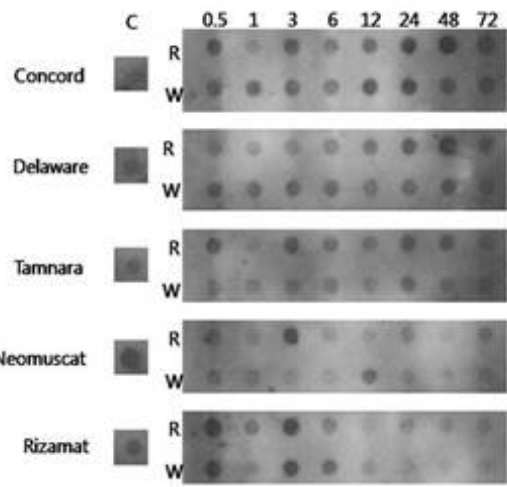

Fig. 5 RNA slot blot hybridization analysis with (A) $\beta$-1,3-glucanase, (B) CHS, (C) LOX, and (D) proline-rich protein as a probe in several grapevine cultivars. C, control; R, R. vitis inoculation; W, wound

A

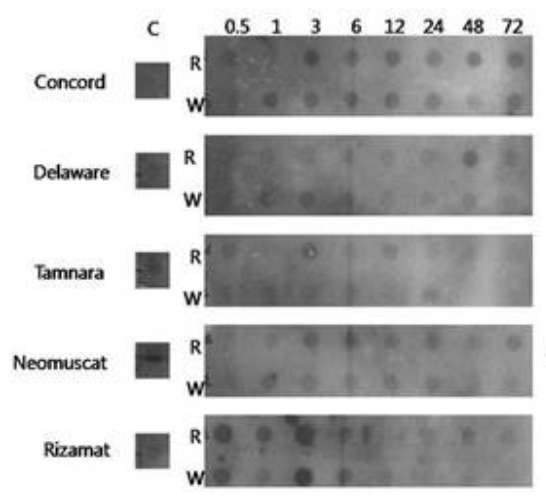

B

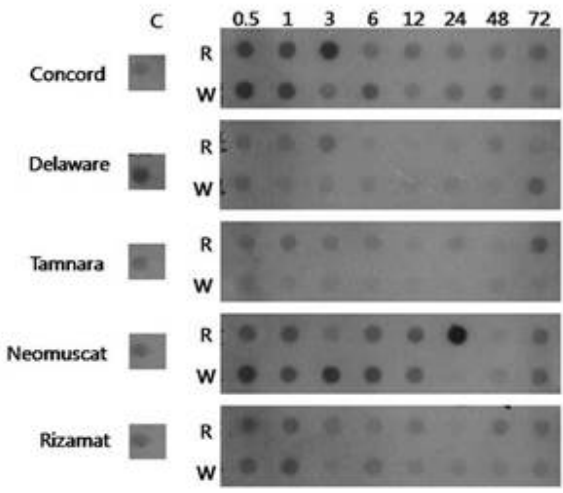

Fig. 6 RNA slot blot hybridization analysis with (A) ATSI 2 and (B) organic cation transporter as a probe in several grapevine cultivars. C, control; R, R. vitis inoculation; W, wound 
Table 5 DEGs responsive to $R$. vitis inoculation, wound, and SA treatment in 'Tamnara' grapevines

\begin{tabular}{|c|c|c|}
\hline & Up-regulated & Down-regulated \\
\hline R. vitis & $\begin{array}{l}\text { ATSI protein } 2 \text { hydrolyzing O-glycosyl compounds } \\
\text { yabby } 15 \text { protein } \\
\text { CHS } \\
\text { Cytochrome P450 } \\
\text { Thaumatin-like protein } \\
\text { GST } \\
\text { Sucrose synthase } \\
\text { Small heat shock protein } \\
\text { LRR containing protein }\end{array}$ & $\begin{array}{l}\text { Expansin } \\
\text { Cytochrome C oxidase polypeptide vc } \\
\text { Seed storage lipid transfer protein } \\
\text { Meiosis } 5 \\
\text { Proline-rich protein apg isolog } \\
\text { SA-induced fragment } 1 \text { protein } \\
\text { WRKY transcription factor } 10 \\
\text { Chitinase-like protein } \\
\text { Zn finger (gata type) family protein }\end{array}$ \\
\hline Wound & - & Fasciclin-like arabinogalactan protein 14 \\
\hline SA & - & $\begin{array}{l}\text { Chloroplast chlorophyll a/b binding protein } \\
\text { ß-Glucanase-like protein } \\
\text { Pollen-specific protein }\end{array}$ \\
\hline R. vitis and $\mathrm{SA}$ & $\begin{array}{l}\text { Btb and taz domain protein } \\
\text { Limonoid UDP-glucosyltransferase } \\
\text { Alkaline a galactosidase }\end{array}$ & $\begin{array}{l}\text { Tonoplast membrane integral protein } 4-4 \\
\text { Histone h3 }\end{array}$ \\
\hline R. vitis and Wound & - & $\begin{array}{l}\text { Cell wall protein } \\
\text { Glucose-methanol-cholineoxidoreductase family protein }\end{array}$ \\
\hline $\begin{array}{l}\text { R. vitis, } \mathrm{SA} \text {, and } \\
\text { Wound }\end{array}$ & $\begin{array}{l}\text { LOX } \\
\text { Aspartyl protease family protein } \\
\text { Heavy metal-associated domain-containing protein } \\
\text { Tyrosine aminotransferase }\end{array}$ & $\begin{array}{l}\text { Lipid transfer protein } \\
\text { Extensin-like protein }\end{array}$ \\
\hline
\end{tabular}

12, and 61 were down-regulated by these treatments, respectively (Table 4).

As shown in the Venn diagram (Fig. 4A), 50 cDNA samples, including ATSI protein 2 hydrolyzing O-glycosyl compounds yabby 15 protein, CHS, cytochrome $\mathrm{P} 450$, thaumatin-like protein, GST, and LRR containing protein, were specifically activated by $R$. vitis inoculation in 'Tamnara' grapevine leaves. However, no cDNA was shown to be up-regulated by wound or SA treatment in grapevines. Five genes, LOX, aspartyl protease family protein, heavy metal-associated domain-containing protein, tyrosine aminotransferase, and one with unknown function, were commonly induced by $R$. vitis inoculation, wound, and SA treatments. Similarly, 1, 4, and 48 cDNAs were down-regulated by wound, SA treatment and $R$. vitis inoculation, respectively (Fig. 4B). Expansin, cytochrome $\mathrm{C}$ oxidase polypeptide vc, seed storage lipid transfer protein, meiosis 5, SA-induced fragment 1 protein, WRKY transcription factor 10, chitinase-like protein, and $\mathrm{Zn}$ finger (gata type) family proteins were specifically downregulated by $R$. vitis inoculation. Four genes were commonly inhibited under both $R$. vitis inoculation and SA treatment, lipid transfer protein, extensin-like protein, and two proteins with unknown function (Table 5). The function of these genes is not clear yet, accordingly, more detailed functional studies should be conducted to determine if they are involved in defense mechanisms.
Comparative analysis of defense-related gene expression in $R$. vitis-inoculated grapevines

To investigate the expression of selected genes, RNA slot blot hybridization was performed using 'Delaware' and 'Concond', 'Neomuscat' and 'Rizamat', and 'Tamnara' grapevine leaves harvested at several time courses. When $\beta$-1,3-glucanase, $C H S, L O X$, proline-rich cell wall protein, ATSI 2, and organic cation transporter genes were used as probes for the hybridization analyses, differential expression was observed between resistant and susceptible cultivars in response to $R$. vitis inoculation and wound treatment (Fig. 5 and 6). The expression patterns of $\beta$-1,3-glucanase, proline-rich protein, and $L O X$ genes in 'Tamnara' grapevines were similar to their expressions in 'Concord' and 'Delaware' which are resistant to crown gall disease. Although proline-rich cell wall protein genes were highly expressed within 12 to $72 \mathrm{~h}$ in 'Concord', 'Delaware', and 'Tamnara' grapevines inoculated with $R$. vitis, they showed low initial expression level, and increased level from $30 \mathrm{~min}$ to $12 \mathrm{~h}$ after $R$. vitis inoculation in 'Neomuscat' and 'Rizamat' grapevines, which are susceptible to crown gall. Conversely, the CHS gene in 'Tamnara' grapevines was expressed at levels similar to those of 'Neomuscat' and 'Rizamat' grapevines. Although transcripts of $C H S$ increased at 48 to $72 \mathrm{~h}$ after $R$. vitis inoculation and wound treatment, they showed low expression at 0.5 to $12 \mathrm{~h}$ after $R$. vitis inoculation in 'Neomuscat', 'Rizamat', and 

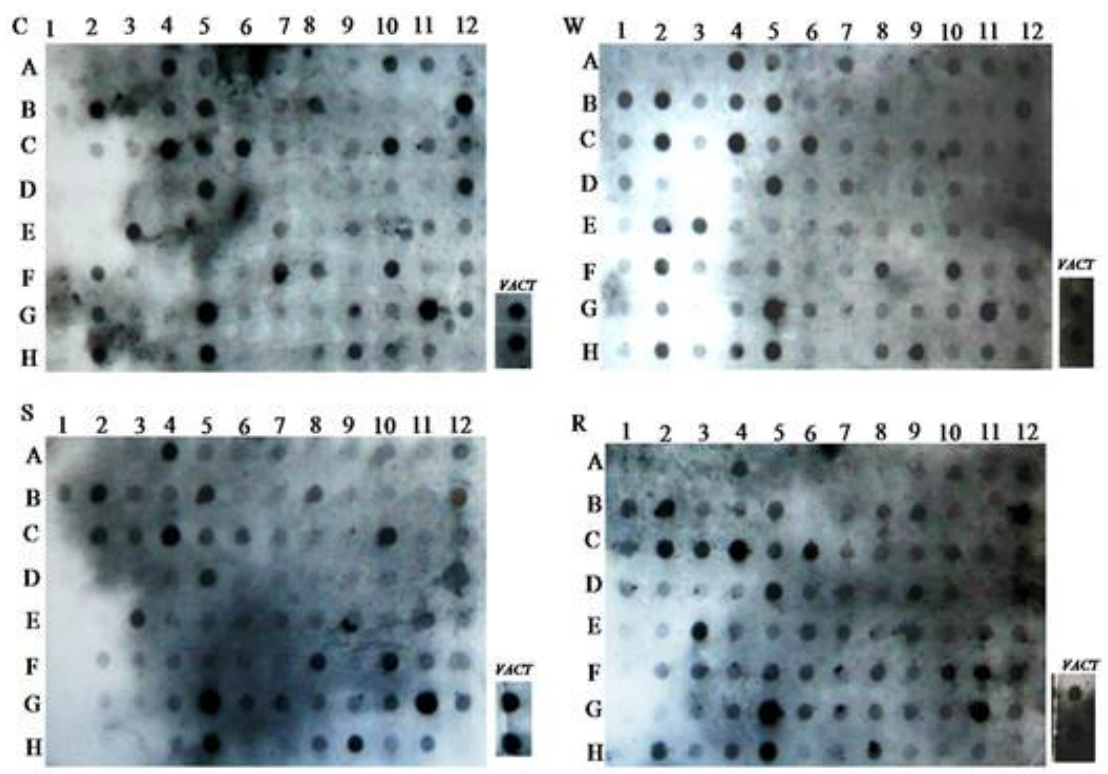

Fig. S1 Slot blot hybridization analysis of 95 highly up-regulated genes in 'Tamnara' grapevines. cDNA probes were synthesized with a ReverTra-plus ${ }^{\mathrm{TM}}$-High Fidelity RT-PCR Kit. C, control; W, wound; S, SA treatment; R, R. vitis inoculation; VACT, actin cloned from 'Tamnara' grapevines
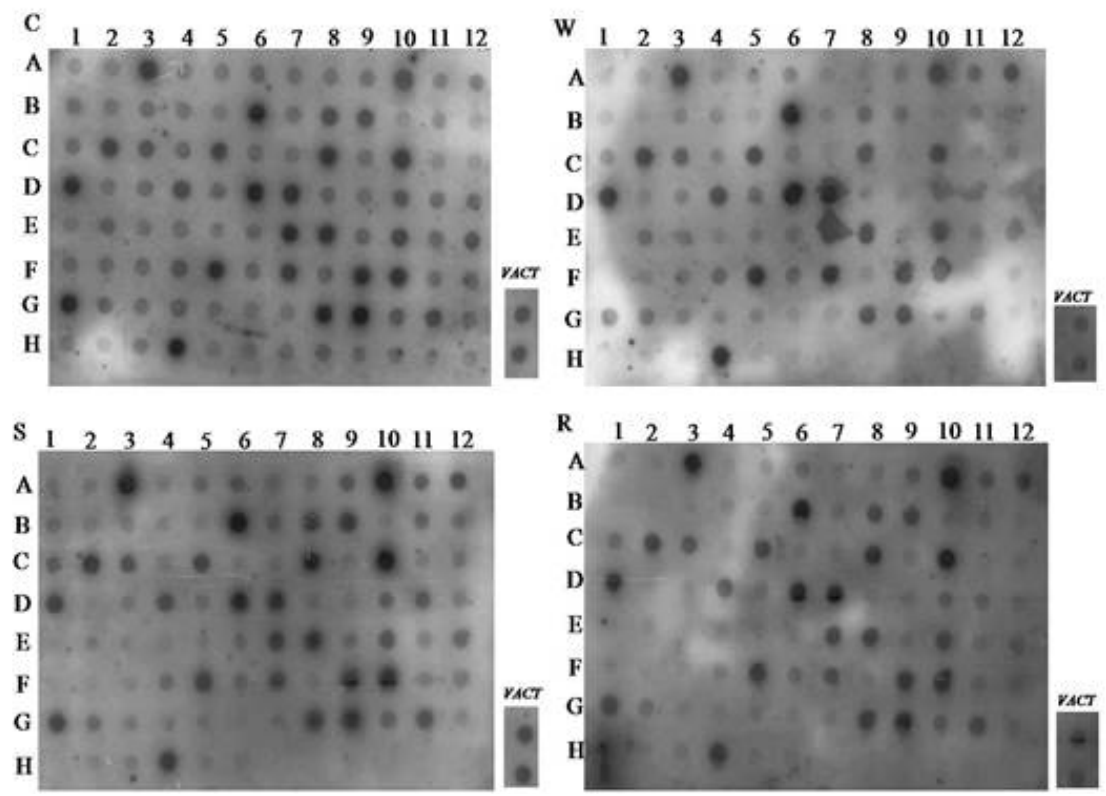

Fig. S2 Slot blot hybridization analysis of 90 highly down-regulated genes in 'Tamnara' grapevines. cDNA probes were synthesized with a ReverTra-plus ${ }^{\mathrm{TM}}$-High Fidelity RT-PCR Kit. C, control; W, wound; S, SA treatment; R, R. vitis inoculation; VACT, actin cloned from 'Tamnara' grapevines

'Tamnara' grapevines. In the case of comparative analysis by Lin et al. (2002), PR genes were expressed in both resistant and susceptible grapevine cultivars in response to $X$. fastidiosa inoculation. These findings suggest that susceptible cultivars have a host defense response mechanism that responds to $X$. fastidiosa inoculation, but they may fail to protect themselves from pathogen infections.

ATSI2 and organic cation transporter genes were highly activated by $R$. vitis inoculation in 'Tamnara' grapevines as confirmed by microarray, RT-PCR, and slot blot hybridization analyses (Fig. 6). ATSI 2, formerly raffinose synthase, is a key enzyme that transfers sucrose into the raffinose oligosaccharide, an oligosaccharide commonly found in plant seeds and other tissues (Nishizawa et al. 2008). This gene is known to be activated by abiotic stressors such as frost, drought, and salt (Kandler and Hopf 1984; Keller and Pharr 
1996; Peterbauer and Richter 2001; Peterbauer et al. 2000). The results of the present study suggest that expression of the ATSI2 gene was also related to the defense responses to $R$. vitis infection in grapevines.

To understand the resistant responses to disease in grapevines, it is important to monitor the specific expression of genes in response pathogen attacks or signal molecules accumulated by pathogens in vines. Among various strategies to screen for specific gene expression, microarray analysis can be used to analyze variations in the expression of thousands of genes simultaneously (Meyers et al. 2004; Schulze and Downward 2001; Stears 2003). In grapevines, microarray analysis has been used in investigations of transcriptomes related to berry development (Deluc et al. 2007; Terrier et al. 2005; Waters et al. 2006), water and salinity stress (Cramer et al. 2007), ultraviolet-B radiation (Pontin et al. 2010), and virus (Espinoza et al. 2007) and fungal infection (Figueiredo et al. 2008).

In this study, the gene expression patterns in response to $R$. vitis bacterium causing crown gall in grapevines were investigated in $R$. vitis-inoculated, salicylic acid (SA)-treated, wounded, and untreated 'Tamnara' grapevines. Microarray analysis using 12,000 gene oligonucleotides of microarray chips constructed with 6,776 unigenes based on EST sequencing revealed that 95 clones were up-regulated by more than 3 times and 90 were down-regulated by more than 5 times in $R$. vitis-inoculated 'Tamnara' grapevines than in untreated vines. Among these, 5 to 61 up-regulated genes, and 10 to 61 clones showed different expression levels in response to wound, SA, and $R$. vitis in grapevines upon RT-PCR and slot blot hybridization analysis. Some genes, such as $\beta$-1,3-glucanase, proline-rich protein, and $L O X$, were induced in moderately resistant cultivars, while others, such as $C H S$, were expressed in moderately resistant and susceptible grapevines in response to $R$. vitis. Further expression studies with various clones per each gene should be conducted to elucidate their roles in resistant responses to pathogens or other stimuli in grapevines. Identification and characterization of the putative genes involved in defense response will be useful for breeding grapes resistant to crown gall. These results could provide a better understanding of the mechanisms of defenses against crown gall disease and clues for identifying new genes that may play a role in defense responses to infection of $R$. vitis.

\section{References}

Anand A, Uppalapati SR, Ryu CM, Allen SN, Kang L, Tang Y, Mysore KS (2008) Salicylic acid and systemic acquired resistantce play a role in attenuating crown gall disease caused by Agrobacterium tumefaciens. Plant Physiol 146:703-715
Benjamini Y, Hochberg Y (1995) Controlling the false discovery rate, a practical and powerful approach to multiple testing. $\mathrm{J}$ Royal Stat Soc Ser B 57:289-300

Burr TJ, Otten L (1999) Crown gall of grape: Biology and disease management. Annu Rev Phytopathol 37:53-80

Chang S, Puryear J, Cairney J (1993) A simple and efficient method for isolating RNA from pine trees. Plant Mol Biol 11:113-116

Cheong YH, Chang H.S., Gupta R., Wang X, Zhu T, Luan S (2002) Transcriptional profiling reveals novel interactions between wounding, pathogen, abiotic stress, and hormonal responses in Arabidopsis. Plant Physiol 129:661-677

Choi, YJ, Yun HK, Park KS, Rho JH, Jeong ST, Lee HJ, Jang HI (2008) Screening genes expressed by Rhizobium vitis inoculation and salicylic acid treatment in grapevines using GeneFishing. J Jpn Soc Hort Sci 77:137-142

Choi, YJ, Yun HK, Park KS, Rho JH, Heo YY, Kim DW, Lee HJ (2010) Transcriptional profiling of ESTs responsive to Rhizobium vitis from 'Tamnara' grapevines (Vitis sp.). J Plant Physiol 167:1084-1092

Cramer GR, Urano K, Delrot S, Pezzotti M, Shinozaki K (2011) Effects of abiotic stress on plants: a systems biology perspective. BMC Plant Biol 11:163

Creelman RA, Mullet JE (1997) Biosynthesis and action of jasmonates in plants. Annu Rev Plant Physiol Plant Mol Biol 48:355-381

Deluc LG, Grimplet J, Wheatley MD, Tillett RL, Quilici DR, Osborne C, Schooley DA, Schlauch KA, Cushman JC, Cramer GR (2007) Transcriptomic and metabolite analyses of Cabernet Sauvignon grape berry development. BMC Genomics $8: 429$

Dong X (1998) SA, JA, ethylene, and disease resistance in plants. Curr Opin Plant Biol 1:316-323

Espinoza C, Vega A, Medina C, Schlauch K, Cramer G, ArceJohnson P (2007) Gene expression associated with compatible viral diseases in grapevine cultivars. Funct Integr Genomics 7:95-110

Figueiredo A, Fortes AM, Ferreira S, Sebastiana M, Choi YH, Sousa L, Acioli-Santos B, Pessoa F, Verpoorte R, Pais MS (2008) Transcriptional and metabolic profiling of grape (Vitis vinifera $\mathrm{L}$.) leaves unravel possible innate resistance against pathogenic fungi. J Exp Bot 59:3371-3381

Hur YY, Jung SM, Yun HK (2015) Current status and prospects of genomics and bioinformatics in grapes. J Plant Biotechnol 42: 298-311

Kandler O, Hopf H (1984) Biosynthesis of oligosaccharides in vascular plants, p. 115-131. In DH Lewis, (ed.). Storage carbohydrates in vascular plants. Cambridge Univ. Press, Cambridge

Keller F, Pharr D (1996) Metabolism of carbohydrates in sinks and sources: Galactosyl-sucrose oligosaccharides, p. 157-183. In E. Zamski, A.A. Schaffer, (eds.). Photoassimilate distribution in plants and crops: Source-sink relationships. Marcel Dekker, New York, USA

Kerr A (1980) Biological control of crown gall through production 
of agrocin 84. Plant Dis 64:28-30

Kunkel BN, Brooks DM (2002) Crosstalk between signaling pathways in pathogen defense. Curr Opin Plant Biol 5:325-331

Lin H, Doddapaneni H, Takahashi T, Walker MA (2007) Comparative analysis of ESTs involved in grape responses to Xylella fastidiosa infection. BMC Plant Biol 7:8

Malamy J, Carr JP, Klessig D, Raskin I (1990) Salicylic acid: A likely endogenous signal in the resistance response of tobacco to viral infection. Science 250:1002-1004

Maleck K, Dietrich RA (1999) Defense on multiple fronts: How do plants cope with diverse enemies? Trends Plant Sci 4:215-219.

Meyers BC, Galbraith DW, Nelson T, Agrawal V (2004) Methods for transcriptional profiling in plants. Be fruitful and replicate. Plant Physiol 135:637-652

Nishizawa A, Yabuta Y, Shigeoka S (2008) Galactinol and raffinose constitute a novel function to protect plants from oxidative damage. Plant Physiol 147:1251-1263

Park KH, Jeong KS, Cha JS (2000) Incidence of severe crown gall disease on tetraploid cultivars of grape in Korea. Plant Pathol J 16:290-293

Park KS, Yun HK, Suh HS, Jeong SB, Cho HM (2004) Breeding of early season grape cultivar 'Tamnara' grapevines (Vitis hybrid) with high quality and disease resistance. Korean J Hort Sci Technol 22:458-461

Peterbauer T, Richter A (2001) Biochemistry and physiology of raffinose family oligosaccharides and galactosyl cyclitols in seeds. Seed Sci Res 11:185-197

Peterbauer T, Mach L, Mucha J, Richter A (2000) Functional expression of a cDNA encoding pea (Pisum sativum L.) raffinose synthase, partial purification of the enzyme from maturing seeds, and steady-state kinetic analysis of raffinose synthesis. Planta 215:839-849

Roh JH, Yun HK, Do KR, Park KS, Choi YJ (2005) Histochemical changes by Agrobacterium vitis infection in grapevines. J Korean Soc Hort Sci 46:260-263

Shah J (2003) The salicylic acid loop in plant defense. Curr Opin Plant Biol 6:365-371

Schulze A, Downward J (2001) Navigating gene expression using microarrays: A technology review. Nature 3:E190-195

Stears RL, Martinsky T, Schena M (2003) Trends in microarray analysis. Nature 9:140-145

Stover EW, Swartz HJ, Burr TJ (1997) Crown gall formation in a diverse collection of Vitis genotypes inoculated with Agrobacterium vitis. Am J Enol Vitic 48:26-32

Terrier N, Glissant D, Grimplet J, Barrieu F, Abbal P, Couture C, Ageorges A, Atanassova R, Léon C, Renaudin JP, et al. (2005) Isogene specific oligo arrays reveal multifaceted changes in gene expression during grape berry (Vitis vinifera L.) development. Planta 222:832-847

The Franch-Italian Public Consortium for Grapevine (2007) The grapevine genome sequence suggests ancestral hexaploidization in major angiosperm phyla. Nature 449:463-467

Van Gelder RN, von Zastrow ME, Yool A, Dement WC, Barchas JD, Eberwine JH (1990) Amplified RNA synthesized from limited quantities of heterogeneous cDNA. Proc Natl Acad Sci USA 87:1663-1667

Waters DL, Holton TA, Ablett EM, Lee LS, Henry RJ (2005) cDNA microarray analysis of developing grape (Vitis vinifera cv. Shiraz) berry skin. Funct Integr Genomics 5:40-58

Yun HK, Rho JH, Park KS, Cha JS, Jeong SB (2003) Screening system for crown gall resistance by pathogen inoculation in grapes. Korean J Hortic Sci Technol 21:325-328 